\title{
Response of neovascular central serous chorioretinopathy to an extended upload of anti-VEGF agents
}

\author{
Benedikt Schworm ${ }^{1}$ (D) Nikolaus Luft ${ }^{1} \cdot$ Leonie F. Keidel $^{1} \cdot$ Felix Hagenau $^{1} \cdot$ Christoph Kern $^{1} \cdot$ Tina Herold $^{1}$. \\ Karsten U. Kortuem ${ }^{1}$. Siegfried G. Priglinger ${ }^{1}$. Jakob Siedlecki ${ }^{1}$
}

Received: 20 November 2019 / Revised: 22 January 2020 / Accepted: 10 February 2020 / Published online: 28 February 2020

(C) The Author(s) 2020

\begin{abstract}
Purpose To determine the anatomical and functional outcomes of an extended 6-month intravitreal anti-vascular endothelial growth factor (anti-VEGF) upload in choroidal neovascularization (CNV) secondary to chronic central serous chorioretinopathy (CSCR).

Methods A retrospective database analysis was performed applying the following inclusion criteria: (1) diagnosis of CSCR, (2) diagnosis of secondary CNV, and (3) treatment of at least six consecutive injections of anti-VEGF. Outcome measures included the change of central retinal subfield thickness, remodeling of the pigment epithelium detachments, and change in visual function.

Results Twenty-one eyes of 21 patients were included. Mean patient age was $65 \pm 8.3$ years, and $35 \%$ of the patients $(n=8)$ were female. Mean disease duration before diagnosis of CNV was $48 \pm 25.3$ months. Mean central retinal thickness decreased from $346 \pm 61$ to $257 \pm 57 \mu \mathrm{m}(p<0.01)$ after the sixth injection while mean visual acuity improved from $0.65 \pm 0.35$ to $0.49 \pm 0.29$ $(\operatorname{logMAR} ; p<0.01)$. Of note, an extended upload of six as opposed to three injections yielded an additional mean central retinal thickness reduction $(280 \pm 46 \mu \mathrm{m}$ vs. $257 \pm 57 \mu \mathrm{m}, p=0.038)$. Significant CNV remodeling was observed as a decrease in pigment epithelium detachment (PED) vertical $(p=0.021)$ and horizontal diameter $(p=0.024)$ as well as PED height $(p<0.01)$. Conclusion An extended anti-VEGF upload of six consecutive injections seems to be effective in inducing CNV remodeling and fluid resorption in CNV complicating chronic CSCR.
\end{abstract}

Keywords Choroidal neovascularization - Central serous chorioretinopathy · Pachychoroid · Pachychoroid neovasculopathy · Anti-VEGF · Pigment epithelium detachment

\section{Introduction}

Choroidal neovascularization (CNV) is a typical complication in chronic central serous chorioretinopathy (CSCR) [1] and represents a major risk factor of poor visual outcome [2]. With central serous chorioretinopathy being a disease of the so-called pachychoroid spectrum [3], secondary CNV in CSCR can also be termed pachychoroid neovasculopathy (PNV) [4]. In these cases, CNV usually develops below the retinal pigment epithelium (RPE) as type $1 \mathrm{CNV}$ [5] and typically manifests on optical coherence tomography as flat irregular pigment epithelium

Benedikt Schworm

benedikt.schworm@med.uni-muenchen.de

1 Department of Ophthalmology, Ludwig-Maximilians-University, Munich, Mathildenstraße 8, 80336 Munich, Germany detachments (PED) [6]. In rare cases, a type $2 \mathrm{CNV}$ (above the $\mathrm{RPE}$ ) can develop additionally. The prevalence of type $1 \mathrm{CNV}$ in CSCR was long time underestimated. Newer data suggest that about one-third of the often observed flat irregular PEDs contain CNV [7]. In a long-term follow-up study of CSCR patients, approximately $24 \%$ developed a secondary CNV after on average 17 years of disease duration [8].

Since the publication of the results of the MINERVA study, intravitreal anti-vascular endothelial growth factor complexation (anti-VEGF) with ranibizumab has been the on-label firstline treatment in secondary CNV due to chronic CSCR [9]. Other treatment options discussed and published include a combined therapy consisting of anti-VEGF and half-fluence photodynamic therapy (hf-PDT) [10] or a full-fluence photodynamic therapy alone [11].

In neovascular AMD, anti-VEGF is routinely administered with an upload of three injections, and further treatment is 
based mainly on the presence of macular fluid and pigment epithelium detachment dynamics. Due to the underlying pathophysiology of pachychoroid disease, the AMD treatment criteria however do not apply to neovascularization due to CSCR, or, as recently termed, PNV. Firstly, PNV usually presents with type $1 \mathrm{CNV}$ [6], which is known to show a weaker response to anti-VEGF compared with type 2 lesions. Secondly, subretinal fluid might be both caused by the underlying pachychoroid and the CNV [12]. Resultingly, as a third caveat, an important treatment effect of anti-VEGF in pachychoroid disease may lie within the induction of choroidal thinning and reduction of choroidal hyperpermeability as opposed to sole fluid reduction [13].

Due to these facts, it is currently unclear whether the effects of anti-VEGF in neovascular CSCR result from CNV remodeling or are mainly caused by choroidal thinning and subretinal fluid resolution. In analogy, it is unclear whether $\mathrm{CNV}$ in central serous chorioretinopathy or PNV in general requires a three-injection upload similar to AMD or might even benefit from an extended upload, especially concerning the anti-VEGF effect on the underlying choroidal thickening and congestion. This study was designed to investigate the anatomical and functional response of neovascular CSCR and the associated CNV to an extended upload of six antiVEGF injections in patients who developed a CNV secondary to CSCR.

\section{Methods}

\section{Participants}

For this retrospective cohort study, the Smart Eye Database of the Department of Ophthalmology of the university hospital, Ludwig-Maximilians-University Munich, Germany, was screened for patients who were treated with anti-VEGF for secondary CNV between January 2016 and December 2018 [14]. The anti-VEGF agents used for the injections were ranibizumab and aflibercept (off-label). Inclusion criteria for this study were the following: (1) diagnosis of central serous chorioretinopathy as a manifestation of pachychoroid disease, (2) diagnosis of $\mathrm{CNV}$ complicating pachychoroid disease (defining PNV), and (3) treatment of at least six consecutive injections of anti-VEGF. Exclusion criteria were confounding comorbidities (diabetic retinopathy, hereditary retinal disease, diseases of the vitreoretinal interface, post-vitrectomized eyes, optic media impeding sufficient image quality) and evidence of polyps or aneurysmal choroidal vessels in indocyanine green angiography (ICGA). This retrospective chart review was conducted according to the regulations of the local ethics committee and the study adhered to the tenets of the Declaration of Helsinki.
Epidemiological data was obtained from each patient, including age, gender, previous ocular comorbidities and procedures, date of first diagnosis of CSCR, anti-VEGF injection dates, number and type of anti-VEGF injections, and corrected visual acuity converted into logarithm of the minimum angle of resolution (LogMAR) at baseline, and each visit for injections until the end of follow-up.

\section{Multimodal imaging}

Multimodal imaging included spectral domain optical coherence tomography (SD-OCT on SPECTRALIS $®$ HRA + OCT, Heidelberg Engineering, Heidelberg, Germany) with acquisition of a volume scan (with and without enhanced-depth imaging mode, EDI $\left.{ }^{\circ}\right)$ at each visit. Central macular thickness (CMT) was assessed in the central 1-mm-diameter circle of the ETDRS grid using the thickness map generated by the Spectralis Software (Heidelberg Eye Explorer 1.9.11.0, Heidelberg, Germany). The vertical subretinal fluid (SRF) diameter was manually measured as the distance between the end of the outer segments and the RPE at the point of maximum fluid accumulation. Subfoveal choroidal thickness (SFCT) was assessed by manually measuring the distance between the Bruch's membrane interface and the choroidalscleral interface under the center of the foveolar depression. Diagnosis of type $1 \mathrm{CNV}$ was obtained by fluorescein angiography (FA) and ICGA (SPECTRALIS® HRA + OCT, Heidelberg Engineering, Heidelberg, Germany) and/or optical coherence tomography angiography (CIRRUS ${ }^{\circledR} 5000$ with Angioplex, Carl Zeiss Meditec, Oberkochen, Germany, and SPECTRALIS ${ }^{\circledR}$ OCT Angiography Module, Heidelberg Engineering, Heidelberg, Germany). In FA and ICGA, features suggestive of neovascularization included neovascular networks in the early phase of FA or ICGA, leakage on FA, and a hyperfluorescent plaque on ICGA in the late phase photographs (Fig. 1). In cases where a clear CNV diagnosis could not be achieved by angiography alone, optical coherence tomography angiography (OCTA) was added to aid in diagnosis.

\section{Anti-VEGF treatment}

All patients received six consecutive injections of anti-VEGF in a 4-weekly delivery scheme. Anti-VEGF agents used were $0.5 \mathrm{mg}$ of ranibizumab (Lucentis ${ }^{\circledR}$; Novartis Pharma AG, Basel, Switzerland) or $2 \mathrm{mg}$ of aflibercept (Eylea; Bayer HealthCare Pharmaceuticals, Berlin, Germany). In cases of non-response after six injections, patients were individually consulted in a shared decision-making manner to either continue the first-line injection regimen or change to second-line options including a switch of the anti-VEGF agent or the addition of a half-dose PDT. 


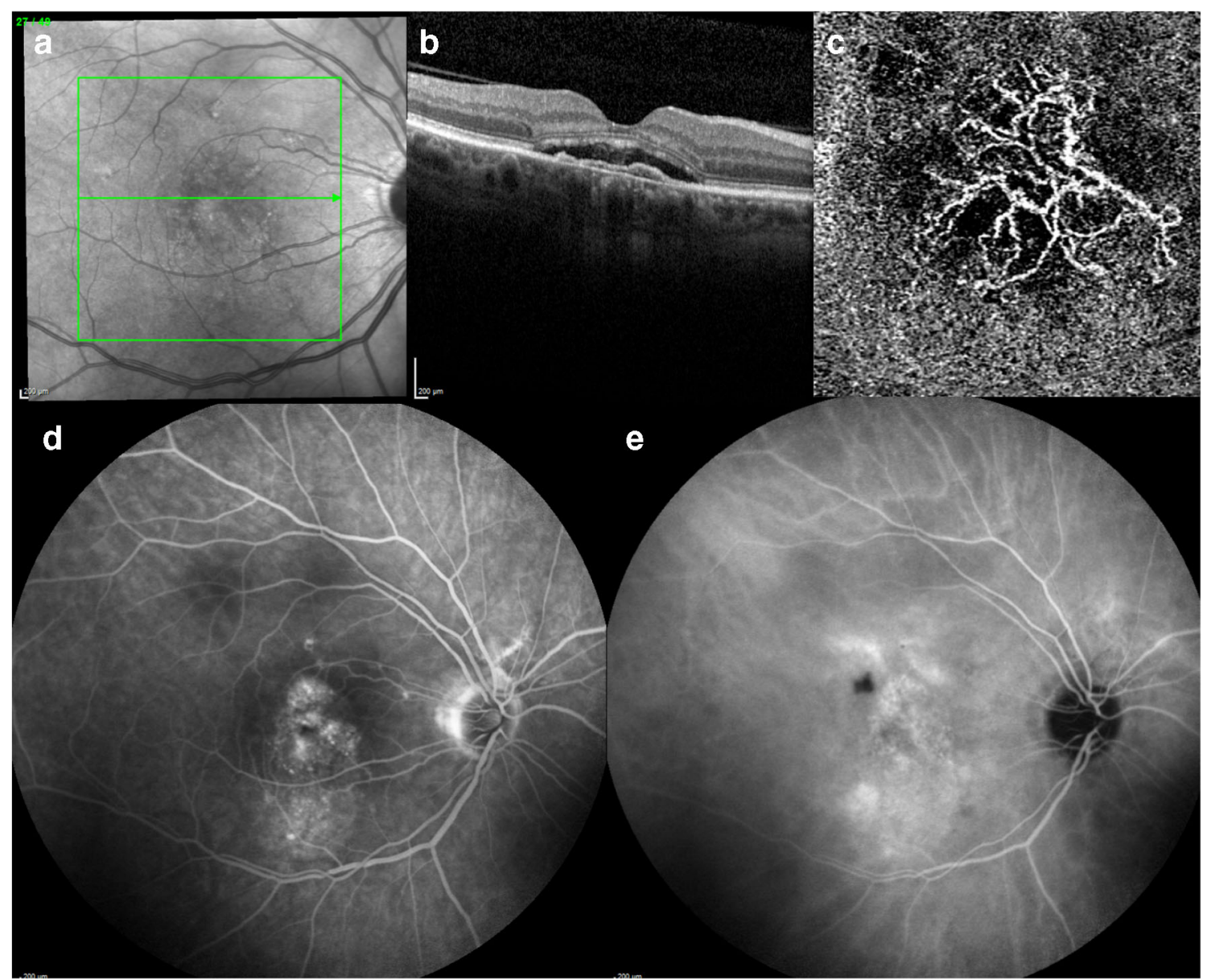

Fig. 1 Example of a 68-year-old patient affected by secondary choroidal neovascularization in chronic central serous chorioretinopathy. a Infrared reflectance. b Horizontal B-scan of optical coherence tomography (OCT) centered to the fovea showing the typical flat irregular pigment epithelium detachment (PED), also referred to as the "double layer sign." c $3 \times 3 \mathrm{~mm}$ enface OCT angiography showing the neovascular network in the PED showed in image b. d Fluorescein angiography image of the macula at 5 min showing granular hyperfluorescence without significant exsudation. e Indocyanine green angiography image of the macular at 5 min showing a discreet plaque sign

\section{Statistical analysis}

All data was collected and preprocessed in Microsoft Excel spreadsheets (version 16.24 for Mac; Microsoft, Redmond, WA, USA). Statistical analysis was performed in SPSS Statistics version 25 (IBM Corp., Armonk, NY, USA). The level to indicate statistical significance was defined as $p<0.05$. The Shapiro-Wilk and KolmogorovSmirnov tests were employed to test for normal distribution of the measured parameters within the study cohort. The paired (samples) $t$ test and the Wilcoxon signed-rank sum test were employed to test for significant differences during follow-up within study eyes depending on the presence of a normal distribution. Repeated measure analysis of variance (ANOVA) was used for comparing measurements at baseline vs. after third injection and after sixth injection. Graphs were plotted in Microsoft Excel and SPSS Statistics version 25 showing the standard deviation.

\section{Results}

\section{Baseline characteristics}

By screening our database of 2498 patients treated with intravitreal injections between January 2016 and December 2018, 140 patients treated with anti-VEGF injections for secondary CNV complicating chronic CSC were identified. Of these, 21 eyes of 21 patients met the inclusion criteria as defined in "Methods." Mean patient age at the first injection was $65 \pm$ 8.3 years (range $48-81$ years), and $35 \%$ of the patients $(n=8)$ were female. Mean disease duration before diagnosis of CNV was $48 \pm 25.3$ months. Four patients $(19 \%)$ had been treated with a half-fluence photodynamic therapy prior to developing the CNV. In one patient (4.8\%), a recent administration of inhaled steroids was documented. In all other patients, there was no evidence of a recent (i.e., in the 6 months before presentation) steroid medication. The diagnosis of CNV was primarily based on the angiography (FA and ICGA) in 
combination with the corresponding OCT image. OCTA was added for initial diagnosis in 17 of the 21 eyes $(81 \%)$ to confirm the diagnosis of a choroidal neovascularization. Regarding the CNV characteristics, 18 eyes (86\%) had a type $1 \mathrm{CNV}$ (between the Bruch membrane and RPE), and three eyes (14\%) had a type $2 \mathrm{CNV}(\mathrm{CNV}$ reaching beyond the $\mathrm{RPE})$. Of the eyes with a type $2 \mathrm{CNV}$, in two cases, the emergence of the type $2 \mathrm{CNV}$ from a type $1 \mathrm{CNV}$ had been observed before initiating treatment, and one case was primarily diagnosed with type $2 \mathrm{CNV}$. Two of the eyes with a type 2 $\mathrm{CNV}$ showed a subretinal hemorrhage on fundoscopy. Mean subfoveal choroidal thickness at baseline was $330 \pm 66 \mu \mathrm{m}$ (range 197-436 $\mu \mathrm{m}$ ). Twelve eyes $(57 \%$ ) had a subfoveal choroidal thickness over $300 \mu \mathrm{m}$.

\section{Anti-VEGF treatment}

All patients received 6-monthly injections. The mean interval between injections was $34 \pm 3$ days. For the first injection, 16 patients received ranibizumab and 5 patients received aflibercept. Two patients were switched from ranibizumab to aflibercept after the third and fourth injection, respectively. No patient was switched from aflibercept to ranibizumab during the first six injections. No adverse events were recorded during the observation period.

\section{Anatomical outcomes}

Mean central retinal thickness (CRT) decreased from $346 \pm$ $61.0(95 \% \mathrm{CI}[319-374 \mu \mathrm{m}])$ to $257 \pm 57.4 \mu \mathrm{m}(p<0.001)$ after the sixth injection (Fig. 2). There was a significant further reduction of mean CRT beyond the third injection (280 \pm $45.6 \mu \mathrm{m}$ vs. $257 \pm 57.4 \mu \mathrm{m}, p<0.001)$ both globally when comparing all three time points in a repeated measure ANOVA as well as specifically when applying a Bonferroni post hoc analysis with pairwise comparisons (BL vs. third

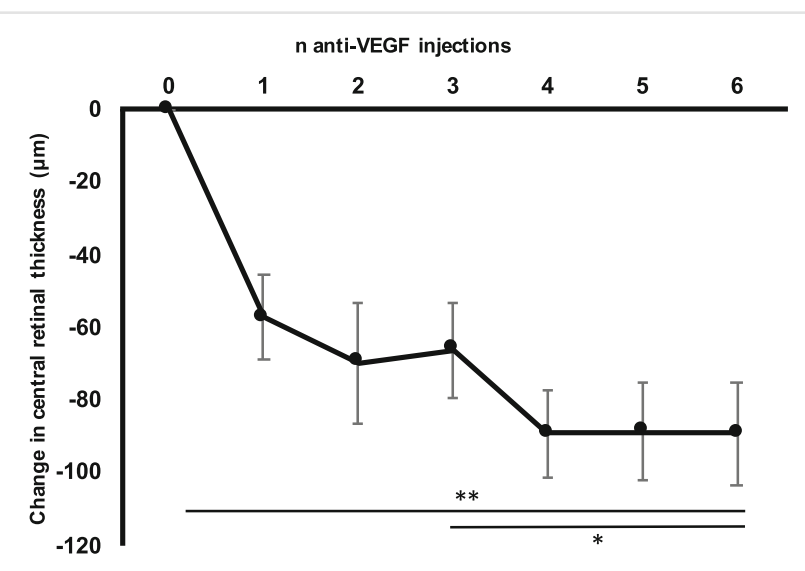

Fig. 2 Change in mean central macular thickness with the number of intravitreal injections. One asterisk indicates statistical significance of $p<0.05$; two asterisks indicate $p<0.01$ injection $p<0.001$, third injection vs. sixth injection $p=$ 0.038). The number of patients with a complete resolution of subretinal fluid improved from 4 (19\%) after the third injection to $11(52.4 \%)$ after the sixth injection. This difference was statistically significant ( $p=0.024$, chi-square test). 19 eyes (90.5\%) had a reduction of CRT after the sixth injection compared with baseline. The remaining two patients $(9.5 \%)$ who had an increase of CRT compared with baseline both showed a recurrence of subretinal fluid under ongoing therapy.

During injection therapy, a marked decrease of pigment epithelium detachment size and thickness was noted (Fig. 3, Table 1). The mean horizontal and vertical diameter of PED decreased from $1980 \pm 543 \mu \mathrm{m}$ and $1861 \pm 658 \mu \mathrm{m}$ to $1794 \pm$ $614 \mu \mathrm{m}$ and $1702 \pm 586.7 \mu \mathrm{m}$, respectively $(p=0.024$ and $p=0.021)$. The horizontal and vertical PED diameter values are for $n=19$ eyes because in two eyes, the boundaries of the PED were not demarcated clearly enough. All other measurements were performed for all $n=21$ eyes. The maximum PED height decreased on average from $169 \pm 77.6$ at baseline to $137 \pm 64.2 \mu \mathrm{m}$ after the third injection $(p=0.01)$ and to $121.6 \pm 56.3 \mu \mathrm{m}(p<0.001)$ after six injections. The

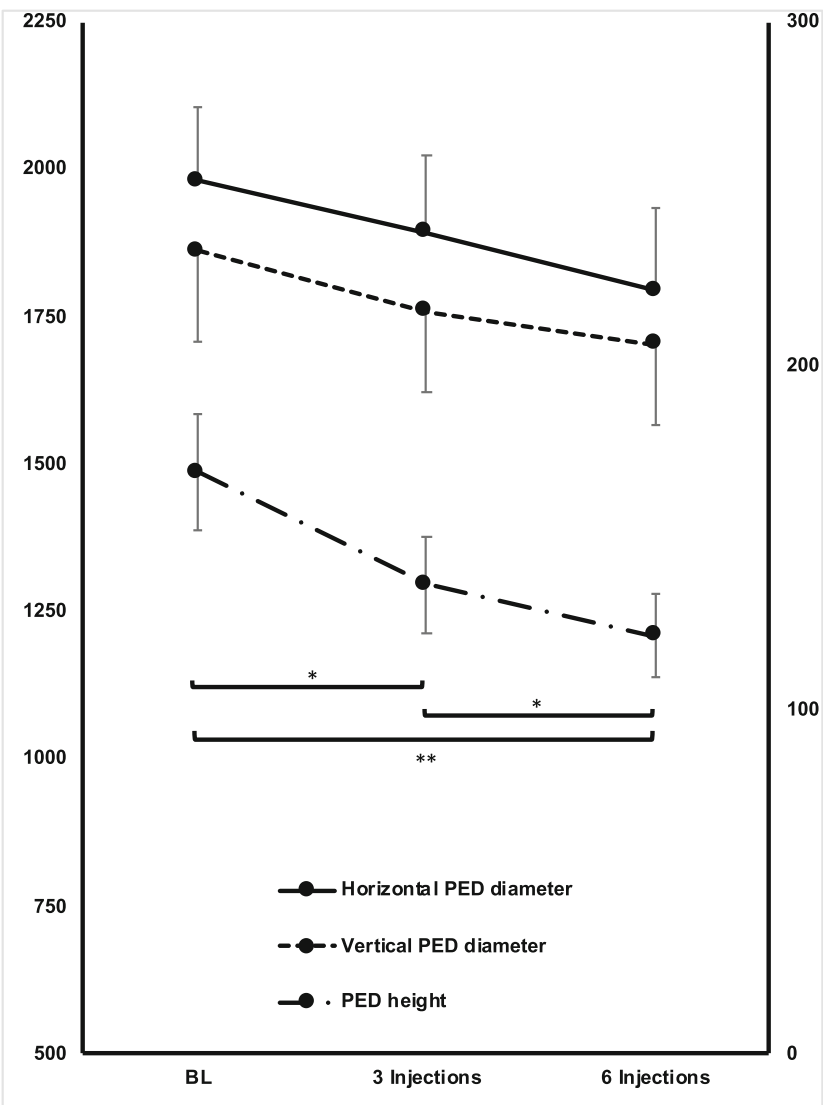

Fig. 3 Mean pigment epithelium detachment measurements. The left $y$ axis indicates the size of vertical and horizontal diameter of the PED; the mean was calculated from $n=19$ measurements. The right $y$-axis indicates the PED height $(n=21)$. All $y$ values are in $\mu \mathrm{m}$, and the error bars indicate the standard error of the mean 
Table 1 Anatomical remodeling under anti-VEGF injection therapy. All values are listed as mean \pm standard deviation. The $p$ value in the last column indicates the statistical significance for the difference between baseline and after six injections. PED, pigment epithelium detachment

\begin{tabular}{lllll}
\hline & Before treatment & After three injections & After six injections & $p$ \\
\hline Mean central retinal thickness $(\mu \mathrm{m})$ & $346 \pm 61.0$ & $280 \pm 45.6$ & $257 \pm 57.4$ & $<0.001$ \\
Mean subfoveal choroidal thickness $(\mu \mathrm{m})$ & $330 \pm 66.4$ & $311 \pm 66.2$ & $302 \pm 69.6$ & $<0.001$ \\
Mean horizontal PED diameter $(\mu \mathrm{m}) *$ & $1980 \pm 543.3$ & $1892 \pm 581.3$ & $1794 \pm 614.0$ & 0.024 \\
Mean vertical PED diameter $(\mu \mathrm{m}) *$ & $1861 \pm 658.1$ & $1760 \pm 592.5$ & $1702 \pm 586.7$ & 0.021 \\
Mean maximum PED height $(\mu \mathrm{m})$ & $169 \pm 77.6$ & $137 \pm 64.2$ & $122 \pm 56.3$ & $<0.001$ \\
Mean vertical SRF diameter $(\mu \mathrm{m})$ & $117 \pm 47.0$ & $58 \pm 47.2$ & $25 \pm 36.5$ & $<0.001$ (Wilcoxon) \\
\hline
\end{tabular}

*The mean values for the PED diameters are for $n=19$; all other values are for $n=21$

difference between PED height after three vs. after six injections continued to be statistically significant $(p=0.029)$. The maximum vertical diameter of the subretinal fluid accumulation decreased on average from $117 \pm 47.0$ at baseline to $25 \pm$ $36.5 \mu \mathrm{m}$ after six injections $(p<0.001$, Wilcoxon signed-rank test). Subfoveal choroidal thickness decreased from an average $330 \pm 66.4$ at baseline to $311 \pm 66.2 \mu \mathrm{m}$ after three injections to $302 \pm 69.6 \mu \mathrm{m}$ after six injections. The difference between all three time points was statistically significant with $p<0.001$. Furthermore, in pairwise comparisons, the difference between baseline and three injections as well as the difference between three injections and six injections was statistically significant with $p<0.001$ and $p=0.03$, respectively.

When arranging those patients who only received ranibizumab (14 eyes) and those who received aflibercept from the beginning or were switched to aflibercept during the extended upload (7 eyes) into subgroups, the results for the reduction of the central retinal thickness were as follows: In the ranibizumab group, a reduction of $-65 \pm 59.2 \mu \mathrm{m}$ was observed while in the aflibercept group, the reduction was $138 \pm 52.5 \mu \mathrm{m}$. The percentage of complete resolution of subretinal fluid was $43 \%(n=6)$ in the ranibizumab group and $71 \%(n=5)$ in the aflibercept group. This difference showed no statistical significance $(p=0.22)$.

\section{Functional outcomes}

Mean visual acuity improved from $0.65 \pm 0.35$ at baseline to $0.51 \pm 0.29$ after the third $(p=0.012)$ and $0.49 \pm 0.29$ $\operatorname{logMAR}(p=0.011)$ after the sixth injection. There was no significant additional increase in visual acuity between the third and sixth injection $(p=0.72)$.

\section{Discussion}

The present study suggests that an extended upload of six consecutive anti-VEGF injections is an effective treatment for choroidal neovascularization secondary to CSCR. CNV membranes secondary to CSCR are known to show a much milder dynamic in contrast to their counterparts in neovascular AMD, myopia, or other secondary causes. For this purpose, this study sought to evaluate the specific responses of $\mathrm{CNV}$ membranes secondary to CSCR. The data of this study suggests that an extended upload with six consecutive anti-VEGF injections is an effective treatment for these lesions. As a new finding, a significant additional benefit of six over three initial injections was observed on central retinal thickness, PED thickness, and PED size.

As recently established by a growing body of evidence, CSCR represents a disease within the new spectrum of "pachychoroid disorders" [3], the exploration of which has been made possible by advances in the visualization of the choroid using enhanced-depth imaging mode on spectral domain OCT. These pachychoroid disorders include pachychoroid pigment epitheliopathy (PPE), defined by characteristic RPE changes without subretinal fluid, pachychoroid neovasculopathy (PNV), defined by the formation of CNV atop of a thickened choroid, and pachychoroid aneurysmal type $1 \mathrm{CNV}$ (pAT1-CNV, also termed polypoidal choroidal vasculopathy, PCV), when formation of aneurysms within the neovascularization occurs. As recently suggested, these four diseases do not represent separate entities, but rather form a continuum of one disease in four stages [15]. Beginning with asymptomatic and uncomplicated choroidal thickening and congestion, the pachychoroid disease then causes characteristic RPE arrosions due to choroidal hyperpermeability, defining PPE as stage 1 disease. The disease can show progress into stage 2 by formation of subretinal fluid, defining CSCR. This stage can become chronic or recurrent and in complicated cases progress to the neovascular stage 3 , defining PNV. The neovascularization can ultimately turn into pachychoroid aneurysmal type $1 \mathrm{CNV}$ (pAT1-CNV/PCV), defined by the formation of CNV aneurysms [16].

When CNV occurs in central serous chorioretinopathy (i.e., pachychoroid neovasculopathy), anti-VEGF injections are the treatment of choice for most ophthalmologists. To date, no standard treatment algorithm for $\mathrm{CNV}$ in central serous chorioretinopathy has been reported. Since the MINERVA study, ranibizumab has been the first-line and on-label option 
for secondary CNV in many different disease conditions, including CSCR [9]. Considering that CSCR is a common disease and $\mathrm{CNV}$ seems to be a natural complication of prolonged disease course [8], there exists only little evidence for treatment.

This study is the first study to describe the effects of a strict upload of six anti-VEGF injections for secondary CNV in CSCR. There have been reports of anti-VEGF treatment outcomes in this disease condition before, albeit without the sixinjection upload. Chablani and colleagues have reported favorable outcomes of anti-VEGF treatment in 46 eyes regarding treatment safety and visual acuity with a mean number of $4.45 \pm 4.1$ anti-VEGF injections during a mean follow-up period of $38.3 \pm 58.9$ months [17]. The mean improvement of visual acuity in their study was $1.16 \pm 3.74$ lines (Snellen) which can be considered more or less comparable with the observed mean improvement of $0.16 \pm 0.26 \log$ MAR in our study. A publication evaluating a combined treatment of antiVEGF injections and half-fluence PDT showed favorable anatomical results with a mean of $1.8 \pm 3.6$ injections over the 12-month follow-up period [10]. Another publication compared full-fluence PDT with intravitreal anti-VEGF agents and reported a more marked decrease in central retinal thickness in the PDT group [11], but it has to be noted that this study included both patients with secondary CNV and polypoidal choroidal vasculopathy. When looking at the MINERVA study, in which only 17 of the 119 patients $(14.3 \%)$ in the treatment arm had a CNV secondary to CSCR, the mean number of ranibizumab injections was 5.8 injections in the ranibizumab arm at month 12 . When comparing anti-VEGF treatment of pachychoroid neovasculopathy with the treatment of neovascular AMD, reports suggest that anti-VEGF treatment is equally effective in both diseases but eyes with pachychoroid neovasculopathy require fewer injections in the maintenance phase of the treatment. However, it has to be noted that these reports partially also included pAT1-CNV/PCV patients [18, 19]. A recently published article compared the effect of the anti-VEGF agents ranibizumab and aflibercept on pachychoroid neovasculopathy [20]. The mean number of injections was 3.5 in the aflibercept group and 4.6 in the ranibizumab group. The results showed that aflibercept had a superior effect on reduction of central retinal thickness and resolution of subretinal fluid compared with ranibizumab. These findings are well in line with those of the present study, in which the patients treated with aflibercept initially or switched to aflibercept showed a more marked decrease in central macular thickness and a higher proportion of dry maculae. It has been discussed that aflibercept might have a stronger effect on choroidal hyperpermeability [21]. This could be explained on the one hand by the additional molecular targets (VEGF-B, PlGF) and on the other hand by a different mechanism of uptake in the tissue [22].
Central serous chorioretinopathy is a disease that commonly occurs in the younger age group. If neovascularization is considered to be a complication of a chronic disease course, it is logical that the average age in this cohort was 65 years. This observation shows good agreement with other publications, in which the mean age ranged between 57 and 68 years $[8,11$, 17, 20].

The pathogenesis of $\mathrm{CNV}$ in pachychoroidal diseases is still not fully understood. In CSCR, the areas of leakage typically lie over areas with choroidal hyperpermeability and dilated choroidal vessels, also named pachyvessels [23]. Flat irregular PEDs containing type $1 \mathrm{CNV}$ usually emerge in these areas of chronically stressed RPE. The role of antiVEGF seems to be different than in AMD as it has been shown that anti-VEGF levels in pachychoroid neovasculopathy are lower than in neovascular AMD [24, 25]. In fact, high baseline anti-VEGF levels seem to be even associated with a poor response to anti-VEGF therapy. In chronic CSCR, proinflammatory cytokines and upregulated angiogenesis are correlated with choroidal vessel abnormalities [26]. Thus, the pathogenetic process leading to and maintaining the CNV is dependent on VEGF and proinflammatory cytokines, but might as well be affected by dysregulation and hyperpermeability of choroidal vessels [12]. However, it is unclear which pathological process is the trigger, choroidal vessel dysregulation or inflammation or a combination of both. Supporting the observation that the mechanism of neovascularization in pachychoroid disease differs strongly from AMD, a recent study suggested that type 1 neovascular membranes in pachychoroid disease contain matured vessels that are less likely to respond to an anti-VEGF treatment [27]. They relate this to a neovascularization pathway that was called arteriogenesis by Spaide in 2015 [28]. As opposed to VEGF-dependent vessel sprouting in AMD-related angiogenesis, arteriogenesis is reported to be induced by shear stress on endothelial stress in small vessels. Shear stress activates mechanotransducers which increase matrixmetalloproteinase activity and recruit inflammatory cells to the extracellular matrix surrounding the vessel. The remodeling process results in expansion of the vessel and thickening of the vessel wall. In our opinion, this theory represents an explanation for the process leading to neovascularization in pachychoroid disease: The increased choroidal perfusion leads to shear stress in the choriocapillaris resulting in the abovementioned extracellular remodeling. This leads to destruction of the Bruch membrane and arrosion of RPE cells (stage 2 in pachychoroid disease, pigment epitheliopathy). As a long-term complication, the arteriogenesis-derived vessels evolve above the Bruch membrane and below the RPE level as a type $1 \mathrm{CNV}$.

For this reason, an important treatment effect of anti-VEGF treatment in pachychoroid disease possibly lies within the induction of choroidal thinning which may be a surrogate 
parameter for the reduction of choroidal hyperpermeability. Studies on the effect of anti-VEGF therapy in nonneovascular CSCR could show that anti-VEGF therapy can lead to less subretinal fluid and induce choroidal thinning [29, 30]. It has to be noted, however, that thinning and reduction of choroidal hyperpermeability might only result from long-term anti-VEGF therapy and could thus not be achieved with an application of only one to three injections. In our collective, a significant reduction of mean subfoveal choroidal thickness was noticeable already after three injections, which, however, was markedly increased after an extended upload of six injections. Furthermore, a significantly higher percentage of "dry macula" was observed after six (52\%) than after three (19\%) injections. The effect of choroidal thinning by a long-term anti-VEGF therapy has been also reported in other studies $[18,20,31]$. Subretinal fluid might be both caused by the underlying pachychoroid and the CNV. Taking this into account as well as the fact that all patients of this collective had CSCR before, it is reasonable that a reduction in choroidal thickness may also lead to less subretinal fluid and thus to a reduced central macular thickness.

Vice versa, another issue that needs further discussion is nonresponse. In our collective, a complete resolution of subretinal fluid was achieved in $52.4 \%$ of the eyes after six injections, meaning that $47.6 \%$ still had significant fluid in spite of half a year of intense anti-VEGF therapy. For nonresponders of anti-VEGF therapy, it has been shown that, analogously to the treatment of pAT1-CNV/ $\mathrm{PCV}$, an adjunctive photodynamic therapy can lead to complete absorption of the subretinal fluid [32]. Moreover, photodynamic therapy has proven to be an effective therapy in non-neovascular CSCR [33, 34]. Therefore, it is pivotal for the understanding of treatment responses that in pachychoroid disorders, subretinal fluid can be both caused by the CNV or the choroidal hyperpermeability. To underline this, it should be mentioned that in our collective, all patients had a decrease in PED thickness. This means that in all patients, the CNV actually responded to the anti-VEGF treatment but not all patients had a subsequent reduction of subretinal fluid. Taking all this into consideration, resolution of subretinal fluid is possibly not the ideal endpoint for a successful treatment of the CNV.

The limitations of the current study are mainly related to the retrospective design and relatively small sample size. Nonetheless, the size of the sample was large enough to apply the intended statistical tests. A limitation regarding multimodal imaging was the fact that data of optical coherence tomography angiography was not sufficient to conduct measurements to all corresponding OCT images and injection time points. Therefore, we had to focus the analysis of anatomical remodeling on OCT-B-scan features. Given the fact that CSCR is often complicated or even caused by the administration of steroid medication, a further limitation of this study lies in the missing data on steroid history. As mentioned above, there was proof of a recent steroid use in one patient. However, it must also be taken into account that many patients after a long course of disease do not remember whether the administration of steroids triggered their first episode. The collective of this analysis represents real-life data. This is why the intended monthly injection interval was not perfectly achieved. The mean interval between the injections of $34.2 \pm$ 3.1 days indicates a good therapy adherence in this collective, taking into account the data from the MINERVA study, in which at 12 months, the mean number of injections in the treatment group was only 5.8 of a possible 10 injections. Due to the non-trial conditions, we decided to measure the endpoints after six injections, not 6 months. Another limitation lies in the fact that there is no 12-month follow-up data to evaluate recurrence rates and the missing evaluation of a maintenance phase. However, this study was designed to evaluate the initial treatment response, and it is the first to evaluate the extended upload of six anti-VEGF injections.

In conclusion, we show that a strict injection regimen for $\mathrm{CNV}$ complicating pachychoroid disease can lead to a favorable anatomical and functional treatment response which is attributable not only to substantial effects of anti-VEGF on VEGF-dependent CNV morphology but also to a modulation of choroidal perfusion. In order to define standard management recommendations, further prospective studies are however necessary.

Funding Information Open Access funding provided by Projekt DEAL.

\section{Compliance with ethical standards}

Conflict of interest Benedikt Schworm received previous speaker honoraria and travel expenses from Novartis Pharma GmbH and Topcon Corporation. Nikolaus Luft received income from honoraria as a lecturer from Alcon Laboratories Inc., NIDEK Co. Ltd and CenterVue SpA. Leonie Keidel received previous speaker fees from Recordati Rare Diseases GmbH. Felix Hagenau has received travel expenses from Pharm-Allergan GmbH. Christoph Kern received travel expenses from Bayer AG. Tina Herold received previous speaker honoraria from Novartis, Pharm-Allergan GmbH, and Bayer AG. Karsten Kortuem received previous speaker honoraria and/or travel expenses from Novartis Pharma GmbH, Bayer AG, and Alcon Pharma GmbH. Karsten Kortuem received personal consultation fees from Big Picture Medical. Karsten Kortuem received travel expenses from Pharm-Allergan $\mathrm{GmbH}$ and Heidelberg Engineering GmbH. Siegfried Priglinger received previous speaker honoraria and/or travel expenses from Novartis Pharma GmbH, Oertli AG, Bayer AG, Alcon Pharma GmbH, and Pharm-Allergan $\mathrm{GmbH}$. Jakob Siedlecki received previous speaker honoraria and travel expenses from Novartis Pharma GmbH, Carl Zeiss Meditec AG, Oculentis OSD Medical GmbH, and Pharm-Allergan GmbH. Jakob Siedlecki received personal consultation fees from Bayer AG.

Ethical approval All procedures performed in studies involving human participants were in accordance with the ethical standards of the LudwigMaximilians-University Munich, Germany, and with the 1964 Helsinki 
Declaration and its later amendments or comparable ethical standards. For this type of study, formal consent is not required. This article does not contain any studies with animals performed by any of the authors.

Open Access This article is licensed under a Creative Commons Attribution 4.0 International License, which permits use, sharing, adaptation, distribution and reproduction in any medium or format, as long as you give appropriate credit to the original author(s) and the source, provide a link to the Creative Commons licence, and indicate if changes were made. The images or other third party material in this article are included in the article's Creative Commons licence, unless indicated otherwise in a credit line to the material. If material is not included in the article's Creative Commons licence and your intended use is not permitted by statutory regulation or exceeds the permitted use, you will need to obtain permission directly from the copyright holder. To view a copy of this licence, visit http://creativecommons.org/licenses/by/4.0/.

\section{References}

1. Spaide RF, Campeas L, Haas A, Yannuzzi LA, Fisher YL, Guyer DR, Slakter JS, Sorenson JA, Orlock DA (1996) Central serous chorioretinopathy in younger and older adults. Ophthalmology 103(12):2070-2079 discussion 2079-2080

2. Loo RH, Scott IU, Flynn HW Jr, Gass JD, Murray TG, Lewis ML, Rosenfeld PJ, Smiddy WE (2002) Factors associated with reduced visual acuity during long-term follow-up of patients with idiopathic central serous chorioretinopathy. Retina 22(1):19-24

3. Gallego-Pinazo R, Dolz-Marco R, Gómez-Ulla F, Mrejen S, Freund KB (2014) Pachychoroid diseases of the macula. Medical hypothesis, discovery \& innovation ophthalmology journal 3(4):111-115

4. Pang CE, Freund KB (2015) Pachychoroid neovasculopathy. Retina 35(1):1-9. https://doi.org/10.1097/iae.0000000000000331

5. Fung AT, Yannuzzi LA, Freund KB (2012) Type 1 (sub-retinal pigment epithelial) neovascularization in central serous chorioretinopathy masquerading as neovascular age-related macular degeneration. Retina 32(9):1829-1837. https://doi.org/10.1097/ IAE.0b013e3182680a66

6. de Carlo TE, Rosenblatt A, Goldstein M, Baumal CR, Loewenstein A, Duker JS (2016) Vascularization of irregular retinal pigment epithelial detachments in chronic central serous chorioretinopathy evaluated with OCT angiography. Ophthalmic Surg Lasers Imaging Retina 47(2):128-133. https://doi.org/10.3928/2325816020160126-05

7. Bousquet E, Bonnin S, Mrejen S, Krivosic V, Tadayoni R, Gaudric A (2018) Optical coherence tomography angiography of flat irregular pigment epithelium detachment in chronic central serous chorioretinopathy. Retina 38(3):629-638. https://doi.org/10.1097/ iae.0000000000001580

8. Mrejen S, Balaratnasingam C, Kaden TR, Bottini A, Dansingani K, Bhavsar KV, Yannuzzi NA, Patel S, Chen KC, Yu S, Stoffels G, Spaide RF, Freund KB, Yannuzzi LA (2019) Long-term visual outcomes and causes of vision loss in chronic central serous chorioretinopathy. Ophthalmology. https://doi.org/10.1016/j. ophtha.2018.12.048

9. Lai TYY, Staurenghi G, Lanzetta P, Holz FG, Melissa Liew SH, Desset-Brethes S, Staines H, Hykin PG (2018) Efficacy and safety of ranibizumab for the treatment of choroidal neovascularization due to uncommon cause: twelve-month results of the MINERVA study. Retina 38(8):1464-1477. https://doi.org/10.1097/iae. 0000000000001744

10. Smretschnig E, Hagen S, Glittenberg C, Ristl R, Krebs I, Binder S, Ansari-Shahrezaei S (2016) Intravitreal anti-vascular endothelial growth factor combined with half-fluence photodynamic therapy for choroidal neovascularization in chronic central serous chorioretinopathy. Eye 30(6):805-811. https://doi.org/10.1038/ eye.2016.41

11. Peiretti E, Caminiti G, Serra R, Querques L, Pertile R, Querques G (2018) Anti-vascular endothelial growth factor therapy versus photodynamic therapy in the treatment of choroidal neovascularization secondary to central serous chorioretinopathy. Retina 38(8):15261532. https://doi.org/10.1097/iae.0000000000001750

12. Cheung CMG, Lee WK, Koizumi H, Dansingani K, Lai TYY, Freund KB (2019) Pachychoroid disease. Eye 33(1):14-33. https://doi.org/10.1038/s41433-018-0158-4

13. Razavi S, Souied EH, Darvizeh F, Querques G (2015) Assessment of choroidal topographic changes by swept-source optical coherence tomography after intravitreal ranibizumab for exudative agerelated macular degeneration. Am J Ophthalmol 160(5):10061013. https://doi.org/10.1016/j.ajo.2015.08.009

14. Kortum KU, Muller M, Kern C, Babenko A, Mayer WJ, Kampik A, Kreutzer TC, Priglinger S, Hirneiss C (2017) Using electronic health records to build an ophthalmologic data warehouse and visualize patients' data. Am J Ophthalmol 178:84-93. https://doi.org/ 10.1016/j.ajo.2017.03.026

15. Siedlecki J, Schworm B, Priglinger SG (2019) The pachychoroid disease spectrum-and the need for a uniform classification system. Ophthalmol Retina 3(12):1013-1015. https://doi.org/10.1016/j. oret.2019.08.002

16. Dansingani KK, Gal-Or O, Sadda SR, Yannuzzi LA, Freund KB (2018) Understanding aneurysmal type 1 neovascularization (polypoidal choroidal vasculopathy): a lesson in the taxonomy of 'expanded spectra'-a review. Clin Exp Ophthalmol 46(2):189-200. https://doi.org/10.1111/ceo.13114

17. Chhablani J, Kozak I, Pichi F, Chenworth M, Berrocal MH, Bedi R, Singh RP, Wu L, Meyerle C, Casella AM, Mansour A, Bashshur Z, Scorza A, Carrai P, Nucci P, Arevalo JF (2015) Outcomes of treatment of choroidal neovascularization associated with central serous chorioretinopathy with intravitreal antiangiogenic agents. Retina 35(12):2489-2497. https://doi.org/10.1097/iae. 0000000000000655

18. Matsumoto H, Hiroe T, Morimoto M, Mimura K, Ito A, Akiyama H (2018) Efficacy of treat-and-extend regimen with aflibercept for pachychoroid neovasculopathy and type 1 neovascular age-related macular degeneration. Jpn J Ophthalmol 62(2):144-150. https:// doi.org/10.1007/s10384-018-0562-0

19. Cho HJ, Jung SH, Cho S, Han JO, Park S, Kim JW (2019) Intravitreal anti-vascular endothelial growth factor treatment for pachychoroid neovasculopathy. J Ocul Pharmacol Ther 35(3): 174-181. https://doi.org/10.1089/jop.2018.0107

20. Jung BJ, Kim JY, Lee JH, Baek J, Lee K, Lee WK (2019) Intravitreal aflibercept and ranibizumab for pachychoroid neovasculopathy. Sci Rep 9(1):2055. https://doi.org/10.1038/ s41598-019-38504-y

21. Hata M, Oishi A, Tsujikawa A, Yamashiro K, Miyake M, Ooto S, Tamura H, Nakanishi H, Takahashi A, Yoshikawa M, Yoshimura N (2014) Efficacy of intravitreal injection of aflibercept in neovascular age-related macular degeneration with or without choroidal vascular hyperpermeability. Invest Ophthalmol Vis Sci 55(12):7874-7880. https://doi.org/10.1167/iovs.14-14610

22. Julien S, Biesemeier A, Taubitz T, Schraermeyer U (2014) Different effects of intravitreally injected ranibizumab and aflibercept on retinal and choroidal tissues of monkey eyes. Br J Ophthalmol 98(6): 813-825. https://doi.org/10.1136/bjophthalmol-2013-304019

23. Dansingani KK, Balaratnasingam C, Naysan J, Freund KB (2016) En face imaging of pachychoroid spectrum disorders with sweptsource optical coherence tomography. Retina 36(3):499-516. https://doi.org/10.1097/iae.0000000000000742

24. Hata M, Yamashiro K, Ooto S, Oishi A, Tamura H, Miyata M, Ueda-Arakawa N, Takahashi A, Tsujikawa A, Yoshimura N 
(2017) Intraocular vascular endothelial growth factor levels in pachychoroid neovasculopathy and neovascular age-related macular degeneration. Invest Ophthalmol Vis Sci 58(1):292-298. https:// doi.org/10.1167/iovs.16-20967

25. Terao N, Koizumi H, Kojima K, Yamagishi T, Yamamoto Y, Yoshii K, Kitazawa K, Hiraga A, Toda M, Kinoshita S, Sotozono C, Hamuro J (2018) Distinct aqueous humour cytokine profiles of patients with pachychoroid neovasculopathy and neovascular agerelated macular degeneration. Sci Rep 8(1):10520. https://doi.org/ 10.1038/s41598-018-28484-w

26. Terao N, Koizumi H, Kojima K, Yamagishi T, Nagata K, Kitazawa K, Yamamoto Y, Yoshii K, Hiraga A, Toda M, Kinoshita S, Sotozono C, Hamuro J (2018) Association of upregulated angiogenic cytokines with choroidal abnormalities in chronic central serous chorioretinopathy. Invest Ophthalmol Vis Sci 59(15):59245931. https://doi.org/10.1167/iovs.18-25517

27. Sacconi R, Tomasso L, Corbelli E, Carnevali A, Querques L, Casati S, Bandello F, Querques G (2019) Early response to the treatment of choroidal neovascularization complicating central serous chorioretinopathy: a OCT-angiography study. Eye. https://doi.org/ 10.1038/s41433-019-0511-2

28. Spaide RF (2015) Optical coherence tomography angiography signs of vascular abnormalization with antiangiogenic therapy for choroidal neovascularization. Am J Ophthalmol 160(1):6-16. https://doi.org/10.1016/j.ajo.2015.04.012

29. Koss MJ, Beger I, Koch FH (2012) Subthreshold diode laser micropulse photocoagulation versus intravitreal injections of bevacizumab in the treatment of central serous chorioretinopathy. Eye 26(2):307-314. https://doi.org/10.1038/eye.2011.282

30. Pitcher JD 3rd, Witkin AJ, DeCroos FC, Ho AC (2015) A prospective pilot study of intravitreal aflibercept for the treatment of chronic central serous chorioretinopathy: the CONTAIN study. Br J Ophthalmol 99(6):848-852. https://doi.org/10.1136/bjophthalmol2014-306018

31. Ting DSW, Yanagi Y, Agrawal R, Teo HY, Seen S, Yeo IYS, Mathur R, Chan CM, Lee SY, Wong EYM, Wong D, Wong TY, Cheung GCM (2017) Choroidal remodeling in age-related macular degeneration and polypoidal choroidal vasculopathy: a 12-month prospective study. Sci Rep 7(1):7868. https://doi.org/10.1038/ s41598-017-08276-4

32. Lee JH, Lee WK (2016) One-year results of adjunctive photodynamic therapy for type 1 neovascularization associated with thickened choroid. Retina 36(5):889-895. https://doi.org/10.1097/iae. 0000000000000809

33. Kretz FT, Beger I, Koch F, Nowomiejska K, Auffarth GU, Koss MJ (2015) Randomized clinical trial to compare micropulse photocoagulation versus half-dose verteporfin photodynamic therapy in the treatment of central serous chorioretinopathy. Ophthalmic Surg Lasers Imaging Retina 46(8):837-843. https://doi.org/10.3928/ 23258160-20150909-08

34. van Dijk EHC, Fauser S, Breukink MB, Blanco-Garavito R, Groenewoud JMM, Keunen JEE, Peters PJH, Dijkman G, Souied EH, MacLaren RE, Querques G, Downes SM, Hoyng CB, Boon CJF (2018) Half-dose photodynamic therapy versus high-density subthreshold micropulse laser treatment in patients with chronic central serous chorioretinopathy: the PLACE trial. Ophthalmology 125(10):1547-1555. https://doi.org/10.1016/j. ophtha.2018.04.021

Publisher's note Springer Nature remains neutral with regard to jurisdictional claims in published maps and institutional affiliations. 